\title{
Terahertz properties of liquid crystals doped with ferroelectric $\mathrm{BaTiO}_{3}$ nanoparticles
}

\author{
U. Chodorow ${ }^{1 *}$, E. Mavrona ${ }^{2,3}$, N. Palka ${ }^{4}$, O. Chojnowska ${ }^{5}$, K. Garbat ${ }^{5}$, S. \\ Saitzek $^{3}$, J.F. Blach ${ }^{3}$, V. Apostolopulos ${ }^{2}$, M. Kaczmarek ${ }^{2}$, J. Parka ${ }^{1,6}$ \\ ${ }^{1}$ Institute of Applied Physics, Military University of Technology, 00-908, Kaliskiego 2, \\ Warsaw, Poland \\ ${ }^{2}$ School of Physics and Astronomy, University of Southampton, Southampton, SO17 1BJ, \\ Southampton, United Kingdom \\ ${ }^{3}$ Université d'Artois, CNRS UMR 8181, Unité de Catalyse et de Chimie du Solide - UCCS, \\ Faculté des Sciences Jean Perrin, SP18, 62307 Lens Cedex, France. \\ ${ }^{4}$ Institute of Optoelectronics, Military University of Technology, 00-908, Kaliskiego 2, \\ Warsaw, Poland \\ ${ }^{5}$ Institute of Chemistry, Military University of Technology, 00-908, Kaliskiego 2, Warsaw, \\ Poland \\ ${ }^{6}$ Institute of Microelectronics and Optoelectronics, Warsaw University of Technology, 00- \\ 665, Nowowiejska 15/19, Warsaw, Poland \\ *Corresponding author: Urszula Chodorow - urszula.chodorow@wat.edu.pl \\ Elena Mavrona-em3e12@soton.ac.uk \\ Norbert Pałka - norbert.palka@wat.edu.pl \\ Olga Chojnowska - olga.chojnowska@wat.edu.pl \\ Katarzyna Garbat - katarzyna.garbat@wat.edu.pl \\ Sebastien Saitzek -sebastien.saitzek@univ-artois.fr \\ Jean Francois Blach - jfrancois.blach@univ-artois.fr \\ Vasilis Apostolopoulos - V.Apostolopoulos@soton.ac.uk \\ Małgorzata Kaczmarek -mfk@soton.ac.uk \\ Janusz Parka-janusz.parka@wat.edu.pl
}




\section{Terahertz properties of liquid crystals doped with ferroelectric $\mathrm{BaTiO}_{3}$ nanoparticles}

In this paper we present the results of the terahertz measurements of liquid crystal ferroelectric $\mathrm{BaTiO}_{3}$ nanoparticles suspensions in the range of frequency from 0.3 up to $3.0 \mathrm{THz}$. Two different sol-gel methods and the harvesting technique were used to fabricate the nanoparticles. Five liquid crystal materials served as hosts for the suspensions: two single compounds: 6CHBT and 2,3',5'-trifluoro-4(4-pentylcyclohexyl)-4'-(trifluoromethoxy)-1,1'-biphenyl, and three mixtures: 1867, 2037 and 2020. We characterize, for the first time, the refractive indices and absorption parameters of suspensions with harvested nanoparticles in the terahertz range and show how the process of the nanoparticles' preparation affects their response. We observed the increase of birefringence for few liquid crystal suspensions in comparison with the pure liquid crystals. The highest increase of birefringence was for 2020 suspension with one kind of ferroelectric nanoparticles. On the other hand in the most cases the addition of ferroelectric nanoparticles to liquid crystal causes the increase of its absorption in the $\mathrm{THz}$ range. The measurements of liquid crystals terahertz properties by using Time-Pulsed Spectrometer were performed.

Key words: liquid crystal, nematic, terahertz spectroscopy, ferroelectric nanoparticles

\section{Introduction}

The doping of a nematic liquid crystal (LC) with a small amount $(<1 \%)$ of ferroelectric nanoparticles (nps) can cause significant changes in their properties such as clearing temperature, threshold voltage, elastic constants, dielectric anisotropy and birefringence in visible range [1-6]. The alternative method for improving or changing such LCs properties is by using chemical synthesis, which is time-consuming and expensive. Additionally, the synthesis of a material with desired physical properties typically requires to use of more than ten components; the more components are included, the more difficult it is to predict final material properties. The ferroelectric nanoparticles can improve the LC materials properties without chemical synthesis and the properties can be varied by changing the type and the amount of nanoparticles mixed with LC $[2,7,8]$. The improved properties of liquid crystals doped with ferroelectric nps are likely to be caused by a strong dipole-dipole interaction 
between the ferroelectric nanoparticles and the surrounding liquid crystal molecules. This dipole-dipole interaction may provide to an increase of the order parameter of LC and thus the dielectric anisotropy and birefringence of the ferroelectric LC suspension. However, the nature of interactions are clearly more complex, as for example, the nps are likely to be charged as well, as recently demonstrated [9].

If the ferroelectric nanoparticles increase the order parameter of LC and thus the birefringence, and also sensitivity on external electric field it can find applications in the $\mathrm{THz}$ frequency range. The improvement in the LCs parameters can help to reduce the thickness of LC layer, which for $\mathrm{THz}$ tends to be quite large, reduce the value of the control voltage and thus improve LC tunability, which is important for the design of the $\mathrm{THz}$ wave modulating devices.

\section{Experimental}

\subsection{Materials}

We chose barium titanate $\left(\mathrm{BaTiO}_{3}\right)$ particles, guided by previous studies of this material, suspended in LCs and because of its ferroelectric properties that found applications in microelectronics and optoelectronics [10-12]. The dielectric properties of $\mathrm{BaTiO}_{3}$ particles are dependent on their crystal structure: the cubic structure exhibits paraelectricity and the tetragonal structure - ferroelectricity. The optimal size of the nanoparticles should be in the range of 10-100 nm. The presence of larger particles may interfere with the homogeneity of the liquid crystal, and thus destroy the orientation of the LC molecules, while the smaller particles lose their ferroelectric properties [7]. Previous work [9,10,13-16] has shown that the preparation of ferroelectric nanoparticles is a difficult process. In recent years, one found new processes for the production of $\mathrm{BaTiO}_{3}$ ferroelectric nanoparticles, including sol-gel method, hydrothermal method, molten salt method, mechanochemical synthesis or combustion 
synthesis $[17,18]$. However, analysis of the data shows that the degree of success of the above processes is significantly different. Resulting powder very often contains large particles, with a wide size distribution, high degree of agglomeration and irregular particles morphology. In this work we used ferroelectric nanoparticles $\mathrm{BaTiO}_{3}$ synthesized by two sol-gel processes as the most promising method. The sol-gel process offers significant advantages such as high purity, chemical homogeneity, particle size control and low process temperature $[12,19]$.

The first sol-gel process we used was based on tetrabutyl titanate $\mathrm{Ti}\left(\mathrm{OC}_{4} \mathrm{H}_{9}\right)_{4}$ and barium acetate $\mathrm{Ba}\left(\mathrm{CH}_{3} \mathrm{COO}\right)_{2}$. The nanoparticles were in solution with heptane, as a solvent, and with oleic acid, as a surfactant. This sample of nanoparticles was labelled as 'Suspension nps3'. Furthermore, the harvesting technique was applied [8]. The harvesting of the ferroelectric single domain nanoparticles, from a large quantity of nanoparticles, is the method where by using electric field gradient ferroelectric particles with the strongest dipole moment are selectively harvested from nanoparticles colloid. Motivation for the harvesting method is to obtain ferroelectric monodomain particles with size smaller than $10 \mathrm{~nm}$. In a liquid harvesting system, a small sealed glass container is fitted internally with a narrow gauge wire axial electrode and an external radial foil electrode. The inner wire electrode is supported within a glass tube. In this way, both the inner and outer electrodes are separated by glass from the fluid harvesting medium to prevent any possibility of direct charge injection into the fluid and to avert any electrolysis. A DC voltage equal $17 \mathrm{kV}$ is applied in the system for the harvesting of the nps. Suspension nps3 was treated in this way and its harvested version is labelled as 'Suspension nps1'. Finally, we used a second sol-gel method for the synthesis of $\mathrm{BaTiO}_{3}$, which is based in n-butoxide titanate $\mathrm{Ti}\left(\mathrm{C}_{8} \mathrm{H}_{17} \mathrm{O}\right)_{4}$ and barium acetate $\mathrm{Ba}\left(\mathrm{CH}_{3} \mathrm{COO}\right)_{2}$ ('Suspension nps2'). The second sol-gel process was used because n-butoxide titanate is around ten times cheaper than tetrabutyl titanate, and thus is more easily accessible. 
The suspensions nps2 and nps3 were characterized by Raman spectroscopy and X-Ray Diffraction (XRD). In the Raman spectra the ferroelectric peak (situated at $305 \mathrm{~cm}^{-1}$ ) was observed, which confirms the nps ferroelectricity. In X-ray patterns, the reflections are characteristic of tetragonal structure which also proves the ferroelectricity of the nps. The average size (measured by the Transmission Electron Microscopy (TEM)) of these nps was between 20 to $80 \mathrm{~nm}[20]$.

We chose two single compounds and three LC mixtures as a host for the ferroelectric nanoparticles: $\quad 6 \mathrm{CHBT}, \quad 2,3^{\prime}, 5^{\prime}$-trifluoro-4-(4-pentylcyclohexyl)-4'-(trifluoromethoxy)-1,1'biphenyl (compound A), 2020, 2037 and 1867. The chemical structures of these LC materials are presented in Table 1.

\section{[insert Table 1 about here]}

The mixture 2020 is composed of ten isothiocyanato compounds with fluorine atoms substituted in lateral position and two, three phenyl rings. Seven compounds possess acetylene bridge group $(\mathrm{C} \equiv \mathrm{C})$ between benzene rings and one compound possess cyclohexane ring.

In total, we prepared eleven liquid crystals suspensions with the ferroelectric nanoparticles obtained with different methods and with different amount of nanoparticles. The ferroelectric nanoparticles suspensions were mixed with the liquid crystal and ultrasonically dispersed for 5-10 minuts to get a homogeneous distribution of the nanoparticles. Then, the heptane was evaporated for about $24 \mathrm{~h}$ at $60-80^{\circ} \mathrm{C}$. The suspensions were ultrasonically mixed several times during this step. The final concentration of the ferroelectric nps is presented in Table 2 . In $[4,5]$ there is a detailed description of the preparation the nematic ferroelectric nps solutions. We used the suspensions nps1, nps2 and nps3 with two materials 6CHBT and 2020. Due to the fact that LCs solutions with nps1 and nps2 hadn't promising properties compare to 
the suspension nps 3 we decided to use only the last one to prepare the solutions with the other LC materials.

\section{[insert Table 2 about here]}

\subsection{Measurements}

Teraview Time-Pulsed Spectrometer 3000 (TPS) [21,22] measurements enabled us to obtain the amplitude and phase of the THz wave after their transition through the sample. The data were also used to obtain absorption and dispersion of the samples. The liquid crystal cells were prepared by using quartz (z-cut) windows of thickness $1.5 \mathrm{~mm}$ and filled with liquid crystals. The copper wires with $0.5 \mathrm{~mm}$ were used both as spacers and as electrodes. To achieve planar alignment of the LC layer, strong AC electric field was applied to the electrodes with frequency $1 \mathrm{kHz}$. For the measured materials, the threshold voltage was $4-8$ $\mathrm{kV} / \mathrm{m}$ and the maximum voltage applied was approximately $30 \mathrm{kV} / \mathrm{m}$. The LC cell was put in the spectrometer chamber with the direction of the $\mathrm{THz}$ beam perpendicular to the cell surface. When the polarization of the $\mathrm{THz}$ wave was parallel or perpendicular to the $\mathrm{LC}$ director, extraordinary or ordinary components of the parameters, such as refractive index and absorption, were measured respectively. As a reference, we used two quartz (z-cut) windows with the thickness $1.5 \mathrm{~mm}$ each.

\section{Results and discussion}

In Figure 1 terahertz parameters of pure and doped 6CHBT liquid crystal are shown. For doped 6CHBT the ordinary refractive indices are higher while the extraordinary refractive indices are lower than for pure 6CHBT. As the results show, the birefringence of $6 \mathrm{CHBT}$ is higher than that for all the ferroelectric suspensions, indicating that the nanoparticles disturb orientation of LC rather than increasing the order parameter. The birefringence for the all 
suspensions differs a little, the highest $\Delta \mathrm{n}$ is obtained for the suspension with nps 3 and the lowest with nps2. The two types of nanoparticles were made through different sol gel processes.

The absorption coefficients for ordinary direction $\alpha_{0}$ are higher for the suspensions as compared with undoped 6CHBT. The absorption coefficient for extraordinary direction $\alpha_{\mathrm{e}}$ for pure 6 CHBT is higher than for nps 2 and nps 3 suspensions, but equal or lower than for nps 1 suspensions. For the all suspensions, however, we observe the decrease in birefringence, indicating perhaps the decrease in the order LC suspension in comparison with the undoped LC sample. If the LC order of the suspensions is indeed decreased, the THz wave could probe both extraordinary and ordinary components of the absorption coefficient. The suspensions with harvested nanoparticles (nps1) are showing a different behavior in comparison with the suspensions without harvesting (nps3). For nps1 suspensions, absorption is higher than for nps 3 in both ordinary and extraordinary directions. It could be argued that the harvested nanoparticles (or the process of harvesting) can introduce additional charges which screen the terahertz waves and thus increase the absorption of the LC solution.

\section{[insert Figure 1 about here]}

In Figure 2, terahertz parameters for pure and doped 2020 liquid crystal are shown. The ordinary refractive indices of the suspensions are comparable to that of for pure 2020 . The extraordinary refractive indices for the nps 1 and nps 2 suspensions are lower and with the nps 3 are higher than for pure LC 2020. The birefringences of suspensions with nps3 are the highest. Since nps3 gave the most promising results, we prepare two suspensions with different concentrations of $0.2 \%$ and $0.5 \%$, respectively by weight. The birefringence of suspension with $0.5 \%$ of nps 3 is lower in the range of frequency $0.3-1.5 \mathrm{THz}$ and higher between $1.5-3.0 \mathrm{THz}$ than for suspension with $0.2 \%$ of nps3. The birefringence for other 
suspensions, namely with nps1 and nps2, respectively, is lower than for pure 2020. In Figure 2e the difference in $\Delta \mathrm{n}$ between the different suspensions and pure 2020 is presented.

In Figure $2 \mathrm{c}$ and $2 \mathrm{~d}$ the absorption coefficients $\alpha_{\mathrm{o}}$ and $\alpha_{\mathrm{e}}$ of 2020 liquid crystal and its suspensions are shown. The ordinary component of absorption for nps 3 suspensions is lower or similar to the absorption of pure 2020 , while the other suspensions have higher absorption than pure 2020 above $0.7 \mathrm{THz}$. The extraordinary component of absorption for pure 2020 is lower than for the all suspensions between 0.7 and $2.5 \mathrm{THz}$. The extraordinary components of the absorption coefficients of all the suspensions have a distinct maximum around 1.51.7 THz, while pure 2020 have only less pronounced maximum around $1.6 \mathrm{THz}$. Additionally, the dichroism of the suspensions with nps3 is higher, while for the other suspensions it is comparable or lower as compare with pure 2020. In suspensions with 2020 we observe the increase of birefringence when there is the increase of dichroism as compare with pure 2020. It may be caused by the increase of the order parameter of the material.

\section{[insert Figure 2 about here]}

As the results from Figures 2, an increase in the birefringence can be achieved with the addition of ferroelectric nanoparticles nps3 to LC 2020. However, the structure and the composition of this material is quite complex, so it not easy to determine the reason of increase in the birefringence.

As the previous studies suggested [5,7], the change in the properties of the nanoparticles suspensions could be related to the ferroelectric nps affecting the order parameter of host LC material. Therefore, we decided to prepare ferroelectric suspensions with LC materials with simpler structure, but composed of compounds similar to LC 2020. First, we investigated single compound, labeled as A, which consists of one type of molecules (Table 1), namely 
those with two phenyl and one cyclohexane rings and three fluorine atoms, substituted in lateral position to benzene rings.

To compound A we added two concentrations of nps3 in stated in Table $2: 0.2 \%$ and $1 \%$ of nanoparticles, by weight. Figure 3 presents terahertz parameters of compound A and its suspensions. We observe a small difference in the birefringence for all the materials, for pure compound A the birefringence is slightly higher than for its suspensions. Between $2 \mathrm{THz}$ and 2.6 $\mathrm{THz}$ birefringence of suspension with $0.2 \%$ of $\mathrm{nps}$ is slightly higher than for pure compound $\mathrm{A}$, but the difference is less than uncertainty of $\Delta \mathrm{n}$.

The extraordinary components of the absorption coefficients for the suspensions have a maximum around $1.5 \mathrm{THz}$ and $2.6 \mathrm{THz}$. For the suspension with the concentrations of $1 \%$ of nps, the absorption is stronger and the maxima more distinct, most likely due to the aggregation of the nanoparticles or some additional, stronger interactions between nanoparticles and surrounding LC molecules. The ordinary absorption coefficients for the suspension with $1 \%$ of nps is comparable, while for the suspension with $0.2 \%$ of nps $\alpha_{0}$ is higher, than for pure compound $\mathrm{A}$ in the range of frequency $0.7-3.0 \mathrm{THz}$. The extraordinary absorption coefficient for the suspension with $0.2 \%$ of nps is comparable, while for the suspension with $1 \%$ of nps $\alpha_{e}$ is higher, than for compound A. For compound A and suspension with $0,2 \%$ of nps $\alpha_{o}>\alpha_{e}$, while for suspension with $1 \%$ of nps it is observed opposite relation $\alpha_{\mathrm{o}}<\alpha_{\mathrm{e}}$ above $1.3 \mathrm{THz}$. Higher concentration of nps increases the probability of nanoparticles agglomeration which can change the absorption of the LC suspension. Additionally we observe a huge increase of $\alpha_{e}$ for the suspension with $1 \%$ of nps which may indicate there were nanoparticles agglomerations.

\section{[insert Figure 3 about here]}


In Figure 4, terahertz properties of mixtures 2037, 1867 and their suspensions are presented. These LC mixtures consist of three kinds of molecules, as shown in Table 1. These LC materials, despite simple composition, have high values of the birefringence, $\Delta \mathrm{n}_{2037}=0.27$ and $\Delta \mathrm{n}_{1867}=0.30$ for $1.5 \mathrm{THz}$, respectively. We observe a slight decrease of birefringence for 2037 ferroelectric LC suspension, in comparison with pure 2037. For pure 1867, the birefringence is lower than for its suspension in the range of frequency $0.3-0.8 \mathrm{THz}$ and above $0.8 \mathrm{THz}$ it is higher.

The extraordinary absorption coefficients $\alpha_{e}$ is comparable to both pure 2037 and its suspension while $\alpha_{\mathrm{o}}$ is higher for the suspension. The ferroelectric nanoparticles suspension with LC 1867 has higher $\alpha_{o}$ and $\alpha_{\mathrm{e}}$ than for pure 1867. For the extraordinary coefficient, there is a wide maximum of absorption around $1.5 \mathrm{THz}$ for LC 1867 suspension, while for pure 1867 the maximum is less pronounced.

\section{[insert Figure 4 about here]}

Mixture 1867 contains similar molecules to compound A and in both cases for ferroelectric suspensions (for compound A with higher concentration of nps) we observe the presence stronger local absorption maximum as compared to pure LC materials for the extraordinary direction. In 2020 suspensions there is also stronger local absorption maximum of $\alpha_{\mathrm{e}}$ as compare to the pure 2020. Some of the molecules components of measured LC materials could increase the probability of these phenomenons for example higher amount of laterally substituted fluorine atoms to compare to the others measured LC materials. The higher amount of laterally substituted F atoms the higher dipole moment of LC molecules [23,24] and thus the dipole-dipole interactions between LC molecules and ferroelectric nanoparticles are stronger and may affect for absorption. 
In Table 3 the relations between absorption coefficients of pure materials and their suspensions are shown. The absorption coefficients $\alpha_{\mathrm{e}}$ for pure materials are comparable or lower than for their ferroelectric suspensions except 6CHBT where $\alpha_{\mathrm{e}}$ for pure material is higher than for its suspensions. Molecules of 6CHBT don't contain fluorine atoms substituted in lateral position, while the other LC materials considered here contain molecules with fluorine atoms. This could be the main reason for the different behavior of $\alpha_{\mathrm{e}}$. Laterally substituted fluorine atoms give a contribution to the polarizability of the molecule along its short axis and thus may lead to different interaction between ferroelectric nps and LC molecules as compared with the materials without laterally substituted polar groups. The absorption coefficient $\alpha_{0}$ for pure LCs are comparable or lower than for their ferroelectric suspensions. In the most cases ferroelectric nanoparticles increase the absorption of LC materials.

\section{[insert Table 3 about here]}

\section{Summary}

To improve LCs properties like clearing temperature, threshold voltage, elastic constants, dielectric anisotropy and birefringence one can use chemical synthesis of new LC materials which is expensive and time-consuming. The other way is doping of LCs with small amount $(<1 \%)$ of ferroelectric nanoparticles. It was shown in many works [1-6] that ferroelectric nanoparticles improve LCs properties in visible range.

In this work terahertz properties of liquid crystal materials doped with ferroelectric $\mathrm{BaTiO}_{3}$ nanoparticles were presented. The best results we noticed for one kind of ferroelectric nanoparticles labelled as nps3. It shows that process of nanoparticles synthesis is very important. There is also difference between suspensions with harvested (nps1) and without harversted (nps3) nanoparticles. We observed higher absorption in the $\mathrm{THz}$ range for $\mathrm{LC}$ 
suspensions with nps1 as compared to suspensions with nps3. The process of harvesting can introduce additional charges which screen the $\mathrm{THz}$ waves and thus increase the absorption of LC solution.

In any case we showed the increase of the LC birefringence in the $\mathrm{THz}$ range is possible by using ferroelectric nanoparticles. The highest increase of the birefringence we observed for 2020 suspension with nps 3 and at the same time the increase of dichroism which might be caused by the increase of the order parameter of the LC. For 2020 suspension with $0.2 \%$ of nps there is the change of $\Delta \mathrm{n}$ equal $4-14 \%$ in the range $0.3-3 \mathrm{THz}$ and with $0.5 \%$ of nps the change is equal $5-10 \%$ to compare to pure 2020 .

\section{Acknowledgments}

This work was supported by the National Centre of Science under Grant PRELUDIUM no. DEC2013/09/N/ST8/01777 and by the DSTL-DGA joint PhD grant DSTLX-100074647.

\section{References}

[1] Ouskova E, Buchnev O, Reshetnyak V, et all. Dielectric relaxation spectroscopy of a nematic liquid crystal doped with ferroelectric $\mathrm{Sn}_{2} \mathrm{P}_{2} \mathrm{~S}_{6}$ nanoparticles. Liq Cryst. 2003;30(10):1325-1239.

[2] Glushchenko A, Cheon Ch.Il, West J, et all. Ferroelectric Particles in liquid Crystals: Recent Frontiers. Mol Cryst Liq Cryst. 2006;453:227-237.

[3] Kaczmarek M, Buchnev O, Nandhakumar I. Ferroelectric nanoparticles in low refractive index liquid crystals for strong electro-optic response. Appl Phys Lett. 2008;92:103307.

[4] Reznikov Yu, Buchnev O, Tereshchenko O, et all. Ferroelectric nematic suspension. Appl Phys Lett. 2003;82(12):1917.

[5] Li F, West J, Glushchenko A,et all. Ferroelectric nanoparticles/liquid-crystal colloids for display applications. J Soc Inf Disp. 2006;14(6):523-527.

[6] Podoliak N, Buchnev O, Henninot M, et all. Elastic constans, viscosity, and response time In nematic liquid Crystals moped with ferro electric nanoparticles. RSC Adv. 2014;4:46068.

[7] Li F, Buchnev O, Cheon Ch.Il, et all. Orientational Coupling Amplification in Ferroelectric Nematic Colloids. Phys Rev Lett. 2006:97:147801.

[8] Cook G, Barnes JL, Basun SA, et all. Harvesting single ferroelectric domain stressed nanoparticles for optical and ferroic applications. J Appl Phys. 2010;108:064309.

[9] Kurochkin O, Mavrona E, Apostolopoulos V, et all. Electrically Charged Dispersions of Ferroelectric Nanoparticles. Appl Phys Lett. 2015;106:043111. 
[10] Corral-Flores V, Bueno-Baques D, Ziolo RF. Synthesis and characterization of novel $\mathrm{CoFe}_{2} \mathrm{O}_{4}-\mathrm{BaTiO}_{3}$ multiferroic core-shell-type nanostructures. Acta Materials. 2010;58:764769.

[11] Li W, Xu Z, Chu R, et all. Structure and electrical properties of $\mathrm{BaTiO}_{3}$ prepared by solgel process. J All Comp. 2009;482:137-140.

[12] Blach JF, Saitzek S, Legrand C, et all. $\mathrm{BaTiO}_{3}$ ferrroelectric nanoparticles dispersed in 5CB nematic liquid crystal: Synthesis and electro-optical characterization. J Appl Phys. 2010;107: 074102.

[13] Atkuri H, Cook G, Evans DR, et all. Preparation of ferroelectric nanoparticles for their use in liquid crystalline colloids. J Opt A: Pure Appl Opt. 2009;11(2):024006.

[14] Qi JQ, Peng T, Hu YM, et all. Direct synthesis of ultrafine tetragonal BaTiO3 nanoparticles at room temperature. Nanosc Res Lett. 2011;6:466.

[15] Morozovska AN, Eliseev EA, Svechnikov GS, et all. Pyroelectric response of ferroelectric nanowires: Size effect and electric energy harvesting. J Appl Phys. 2010;108:042009.

[16] Cook G, Reshetnyak Yu, Ziolo RF, et all. Asymmetric Freedericksz transitions from symmetric liquid crystal cells doped with harvested ferroelectric nanoparticles. Opt Exp. 2010;18(16):17339-17345.

[17] Sahoo GK. Synthesis and characterization of BaTiO3 prepared by molten salt synthesis method [master's thesis], Rourkela - 769 008: Department of Ceramic Engineering, National Institute of Technology; 2009.

[18] Wu YT, Wang XF, Yu ChL, et all. Preparation and characterization of barium titanate $\left(\mathrm{BaTiO}_{3}\right)$ nano-powders by Pechini sol-gel method. Mat Manuf Proc. 2012;27:1329-1333.

[19] Kobayashi Y, Nishikata A, Tanase T, et all. Size effect on crystal structures of barium titanate nanoparticles prepared by sol-gel method. J Sol-Gel Sc Tech. 2004;29:49-55.

[20] Mavrona E, Chodorow U, Barnes ME, et all. Refractive indices and birefringence of hybryd liquid crystals - nanoparticles composite materials in the terahertz region. AIP Adv. 2015;5(7):077143.

[21] Palka N. Identification of concealed materials, including explosives, by terahertz reflection spectroscopy. Opt Eng. 2014;53:031202.

[22] Chodorow U, Parka J, Garbat K. Spectral and Photorefractive Properties of Nematic Liquid Crystals from the CHBT Family in the THz Range. Liq Cryst. 2013;40:1089-1094.

[23] Dąbrowski R, Kula P, Herman J. High birefringence liquid crystal. Crystals. 2013;3:443482.

[24] Kula P, Aptacy A, Herman J, et all. The synthesis and properties of fluoro-substituted analogues of 4-butyl-4'-[(4-butylphenyl)ethynyl]biphenyls. Liq Cryst. 2013;40(4):482-491. 
Table 1. The chemical structures of measured liquid crystal materials a) 6CHBT, b) compound A, c) 2037, d) 1867.

Table 2. The liquid crystal ferroelectric nanoparticles solutions.

Table 3. The relations between absorption coefficients of pure LCs and their suspensions with ferroelectric nanoparticles nps3 in measured $\mathrm{THz}$ range.

Figure 1. Terahertz properties of 6CHBT and its ferroelectric suspensions a) refractive indices, b) birefringence, c) ordinary and d) extraordinary absorption coefficients.

Figure 2. Terahertz properties of 2020 and its ferroelectric suspensions a) refractive indices, b) birefringence, c) ordinary and d) extraordinary absorption coefficients, e) difference of birefringence.

Figure 3. Terahertz properties of compound A and its ferroelectric suspensions a) refractive indices, b) birefringence, c) absorption coefficients, d) difference of birefringence.

Figure 4. Terahertz properties of 2037 and 1867 and their ferroelectric suspensions a,d) refractive indices, $b, e$ ) birefringence, $c, f)$ absorption coefficients. 
Table 1. The chemical structures of measured liquid crystal materials a) 6CHBT, b) compound A, c) 2037, d) 1867.

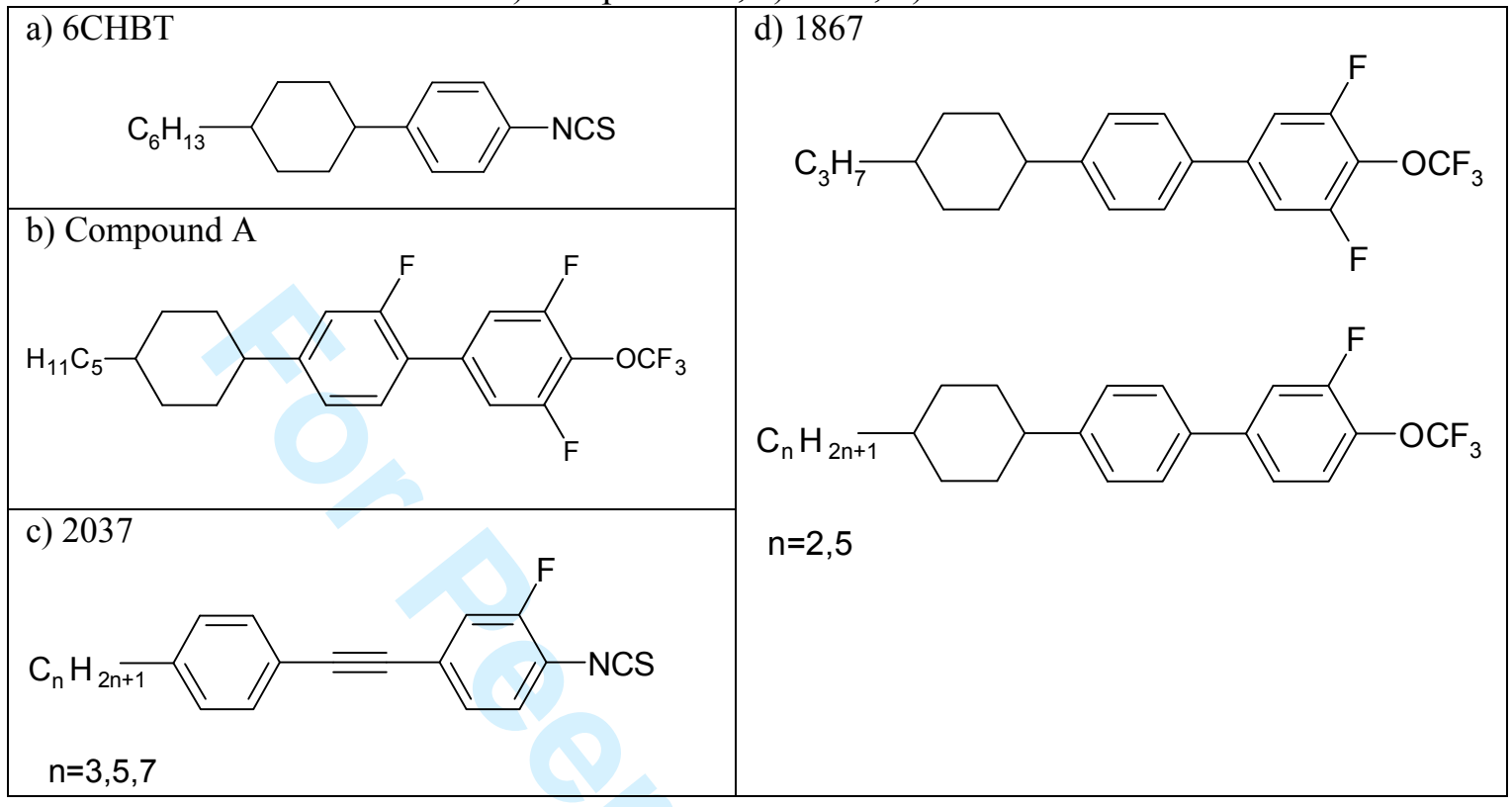

Table 2. The liquid crystal ferroelectric nanoparticles solutions.

\begin{tabular}{|c|c|c|c|}
\hline LC nps & $n p s 1[\mathrm{w} / \mathrm{w}]$ & $\mathrm{nps} 2[\mathrm{w} / \mathrm{w}]$ & $\mathrm{nps} 3[\mathrm{w} / \mathrm{w}]$ \\
\hline 6 CHBT & $0.2 \%$ & $0.2 \%$ & $0.2 \%$ \\
\hline \multirow{2}{*}{2020} & $0.2 \%$ & $0.2 \%$ & $\begin{array}{l}0.2 \% \\
0.5 \%\end{array}$ \\
\hline Compound A & & & $\begin{array}{l}0.2 \% \\
1.0 \%\end{array}$ \\
\hline 2037 & & $0.2 \%$ \\
\hline 1867 & & & $0.2 \%$ \\
\hline
\end{tabular}

Table 2. The relations between absorption coefficients of pure LCs and their suspensions with ferroelectric nanoparticles nps3 in measured $\mathrm{THz}$ range.

\begin{tabular}{|c|c|c|c|c|c|}
\hline amount of nps3 & 6CHBT & 2020 & Compound A & 2037 & 1867 \\
\hline $0.2 \%$ & $\begin{array}{c}\alpha_{\mathrm{op}}<\alpha_{\mathrm{os}} \\
\alpha_{\mathrm{ep}}>\alpha_{\mathrm{es}}\end{array}$ & $\begin{array}{c}\alpha_{\mathrm{op}} \approx>\alpha_{\mathrm{os}} \\
\alpha_{\mathrm{ep}}<\alpha_{\mathrm{es}}\end{array}$ & $\begin{array}{c}\alpha_{\mathrm{op}}<\alpha_{\mathrm{os}} \\
\alpha_{\mathrm{ep}} \approx \alpha_{\mathrm{es}}\end{array}$ & $\begin{array}{c}\alpha_{\mathrm{op}}<\alpha_{\mathrm{os}} \\
\alpha_{\mathrm{ep}} \approx \alpha_{\mathrm{es}}\end{array}$ & $\begin{array}{c}\alpha_{\mathrm{op}}<\alpha_{\mathrm{os}} \\
\alpha_{\mathrm{ep}}<\alpha_{\mathrm{es}}\end{array}$ \\
\hline $0.5 \%$ & $\begin{array}{c}\alpha_{\mathrm{op}} \approx \alpha_{\mathrm{os}} \\
\alpha_{\mathrm{ep}}<\alpha_{\mathrm{es}}\end{array}$ & $\begin{array}{c}\alpha_{\mathrm{op}} \approx \alpha_{\mathrm{os}} \\
\alpha_{\mathrm{ep}}<\alpha_{\mathrm{es}}\end{array}$ \\
\hline
\end{tabular}

*p - pure LC, s - ferroelectric nanoparticles LC suspension 


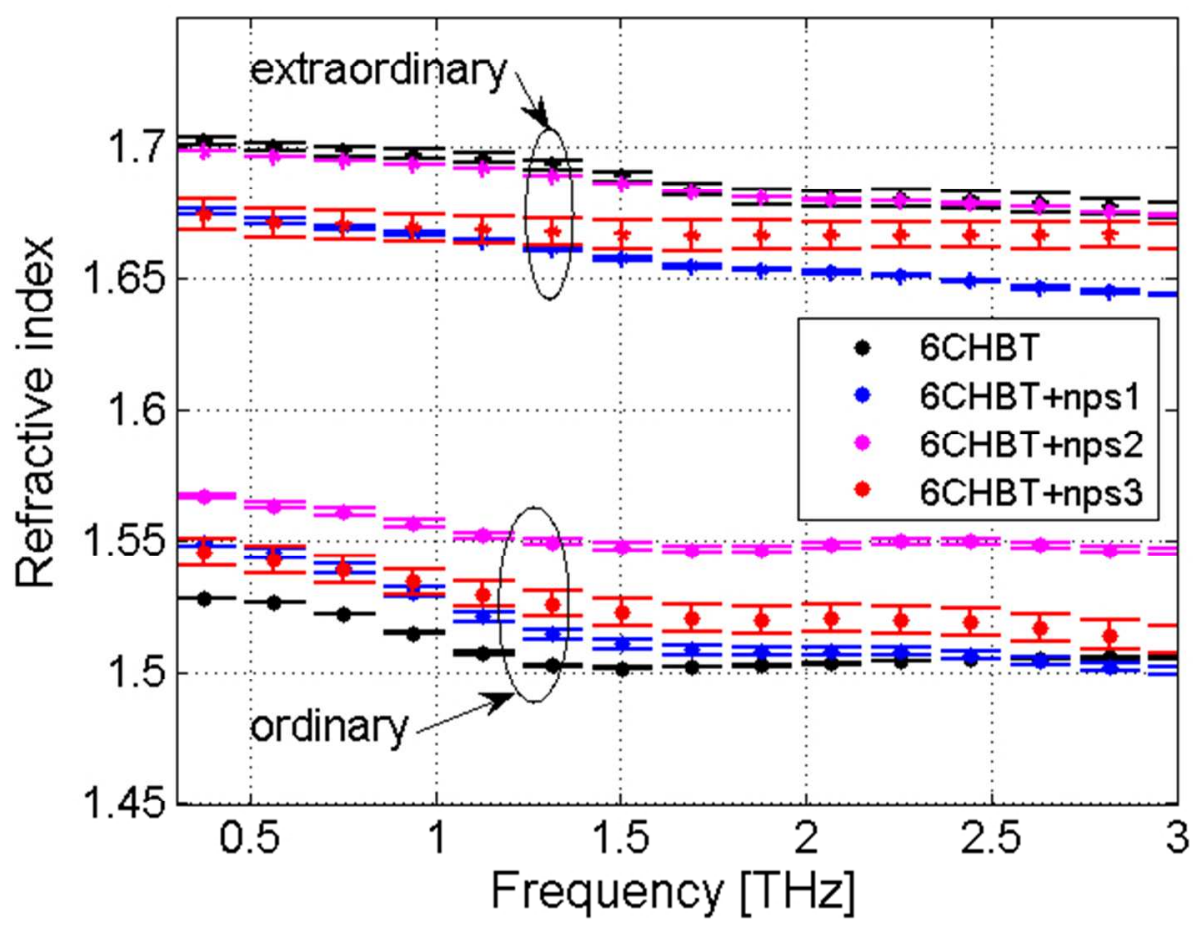

Figure 1a. Refractive indices of 6CHBT and its ferroelectric suspensions. $147 \times 111 \mathrm{~mm}(116 \times 116 \mathrm{DPI})$ 


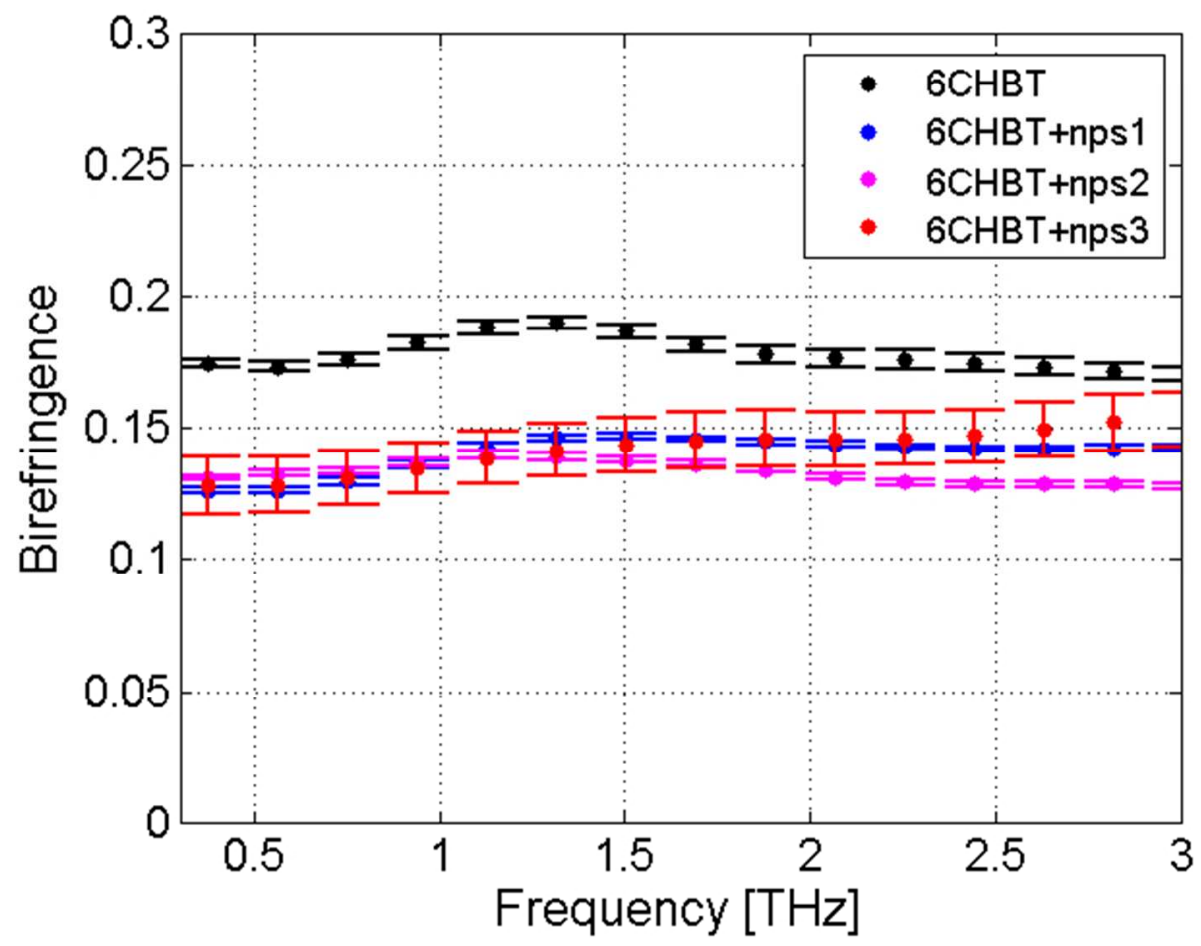

Figure 1b. Birefringence of $6 \mathrm{CHBT}$ and its ferroelectric suspensions. $147 \times 111 \mathrm{~mm}(116 \times 116 \mathrm{DPI})$ 


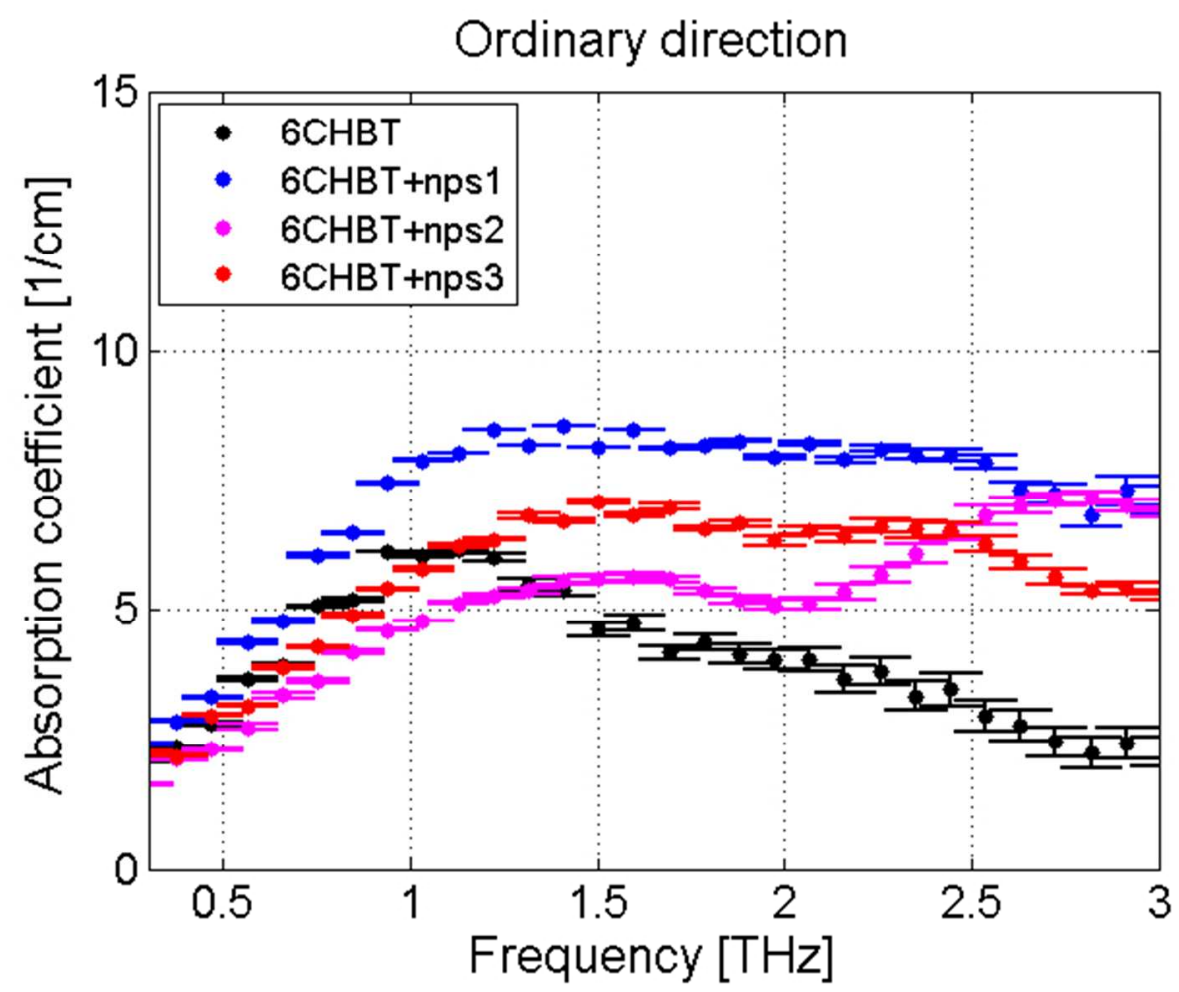

Figure 1c. Ordinary absorption coefficients of 6CHBT and its ferroelectric suspensions. $147 \times 111 \mathrm{~mm}(116 \times 116 \mathrm{DPI})$ 


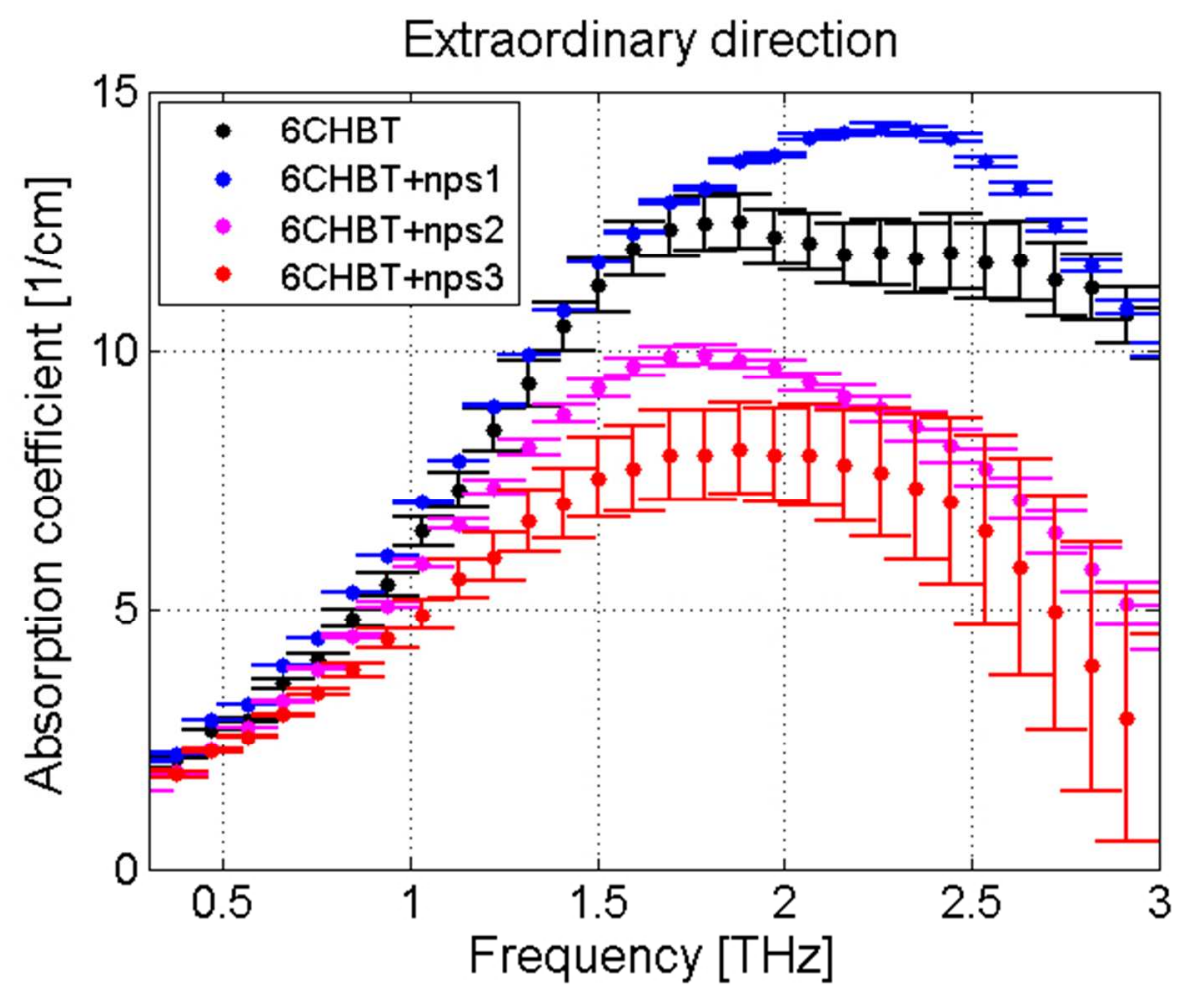

Figure 1d. Extraordinary absorption coefficients of $6 \mathrm{CHBT}$ and its ferroelectric suspensions. $147 \times 111 \mathrm{~mm}(116 \times 116 \mathrm{DPI})$ 


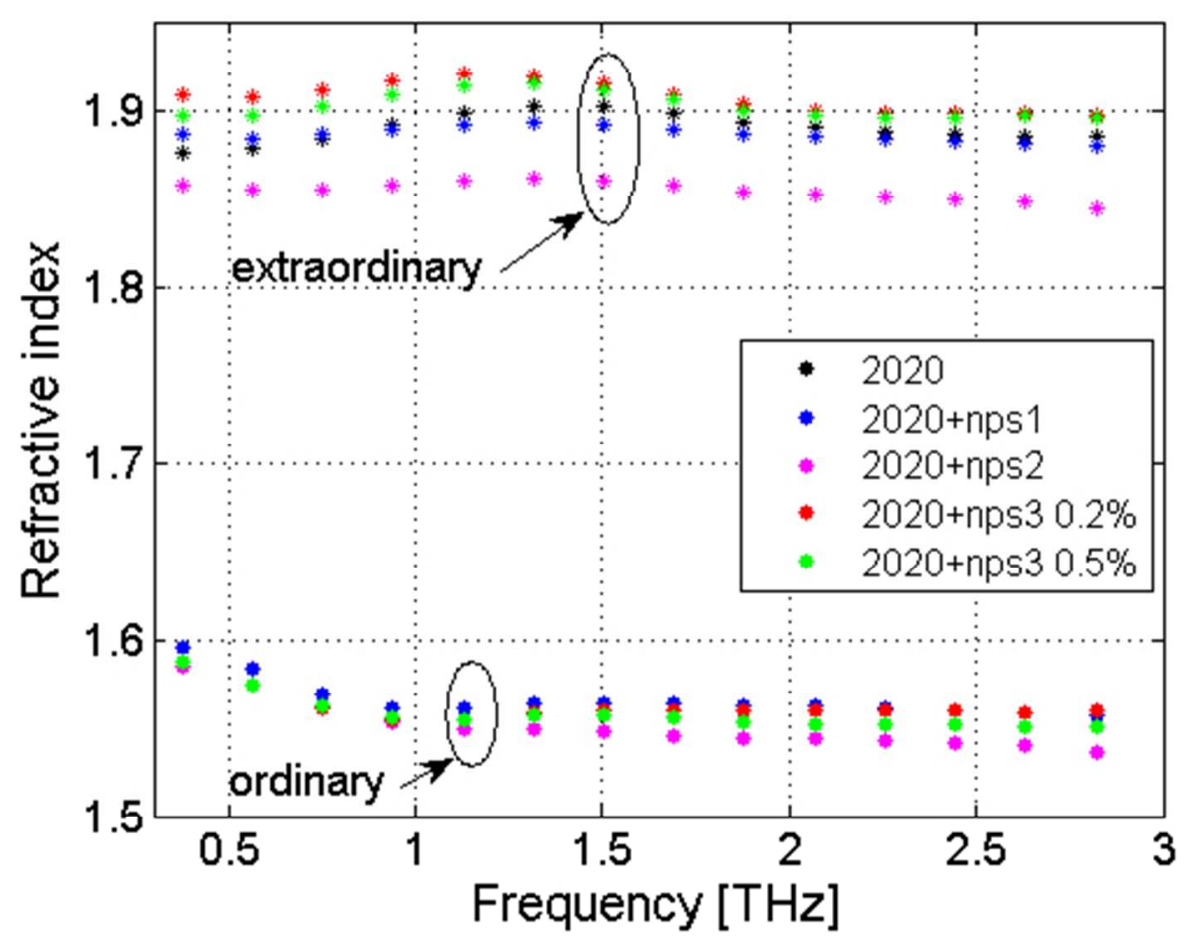

Figure 2a. Refractive indices of 2020 and its ferroelectric suspensions.

$148 \times 112 \mathrm{~mm}(96 \times 96 \mathrm{DPI})$ 


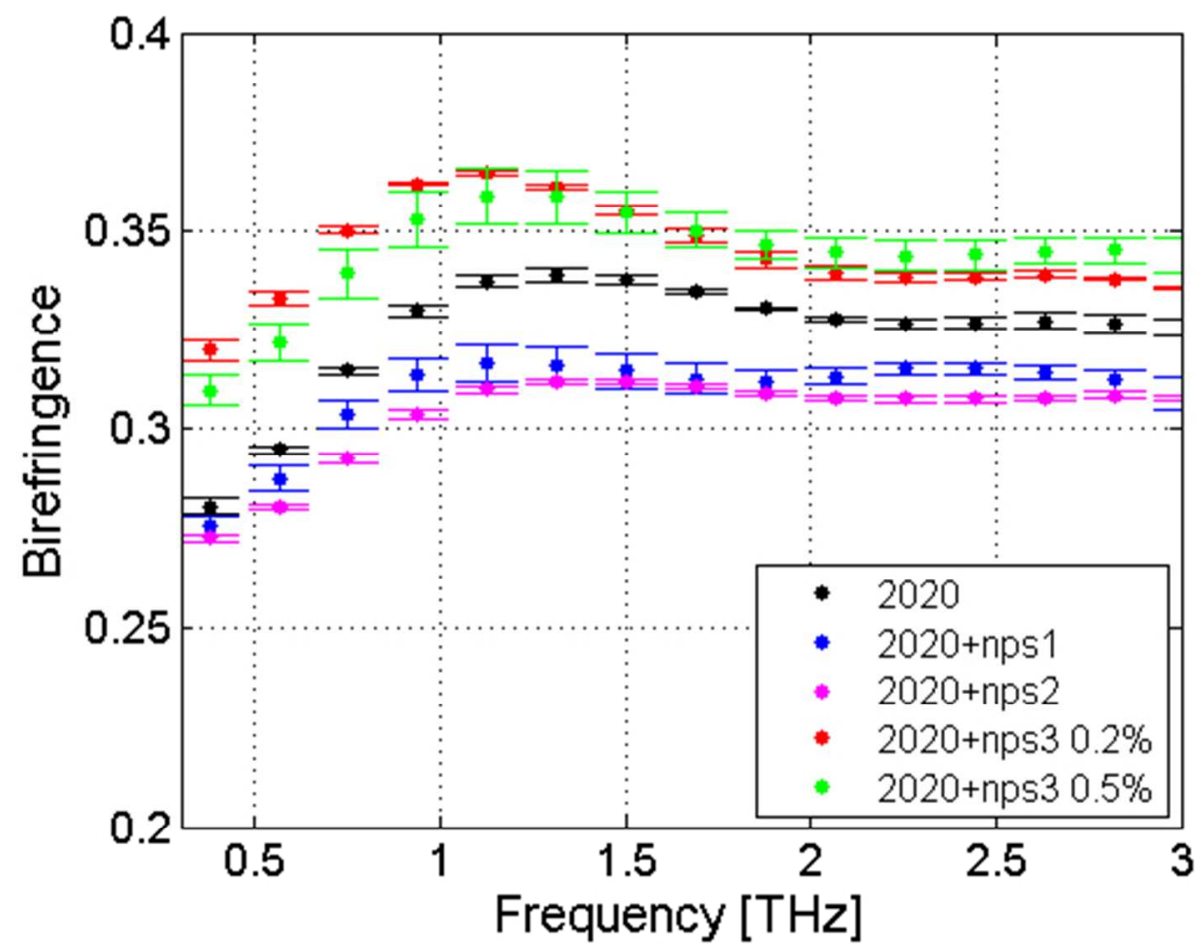

Figure 2b. Birefringence of 2020 and its ferroelectric suspensions.

$148 \times 112 \mathrm{~mm}(96 \times 96 \mathrm{DPI})$ 


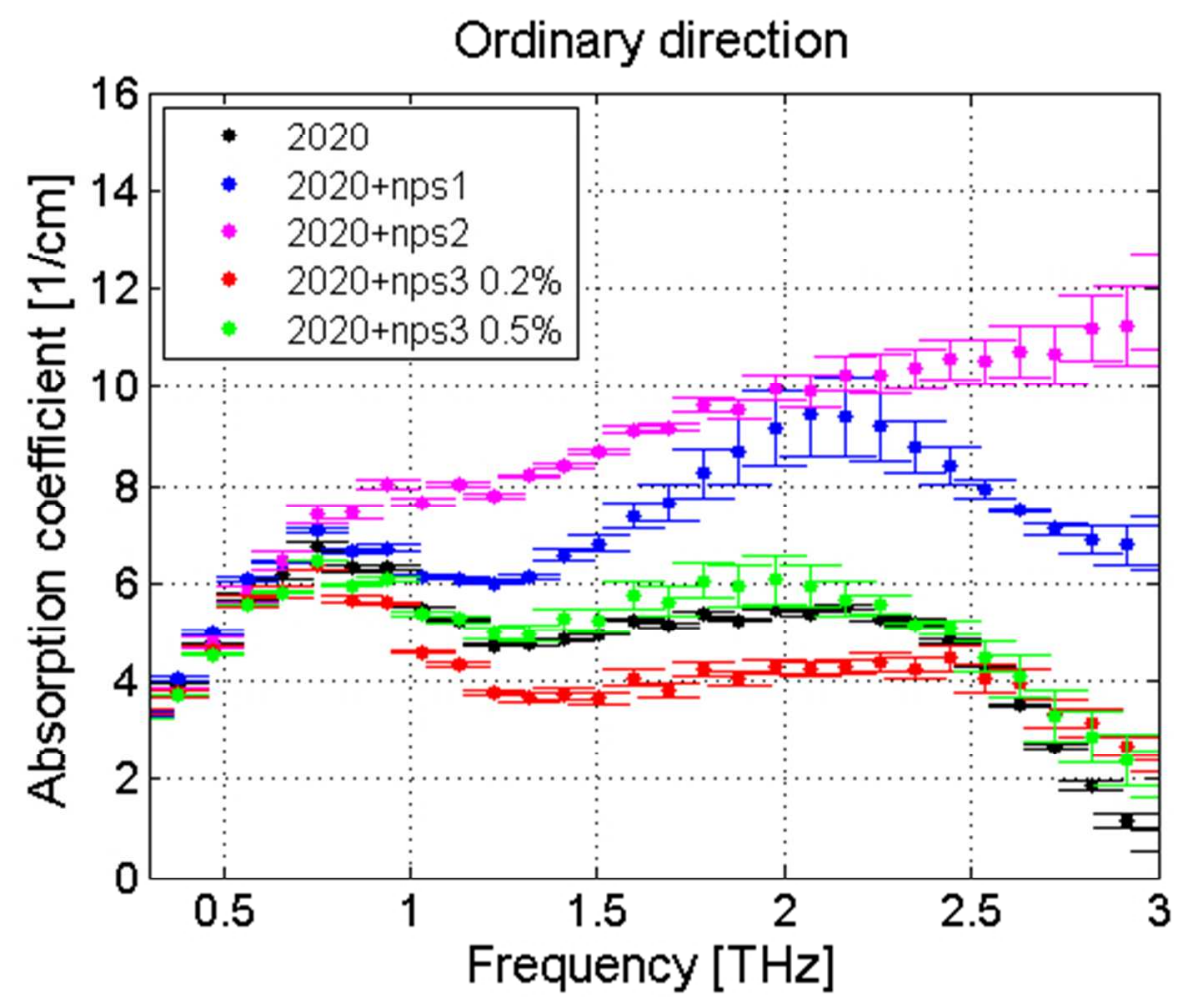

Figure 2c. Ordinary absorption coefficients of 2020 and its ferroelectric suspensions.

$148 \times 112 \mathrm{~mm}(96 \times 96 \mathrm{DPI})$ 


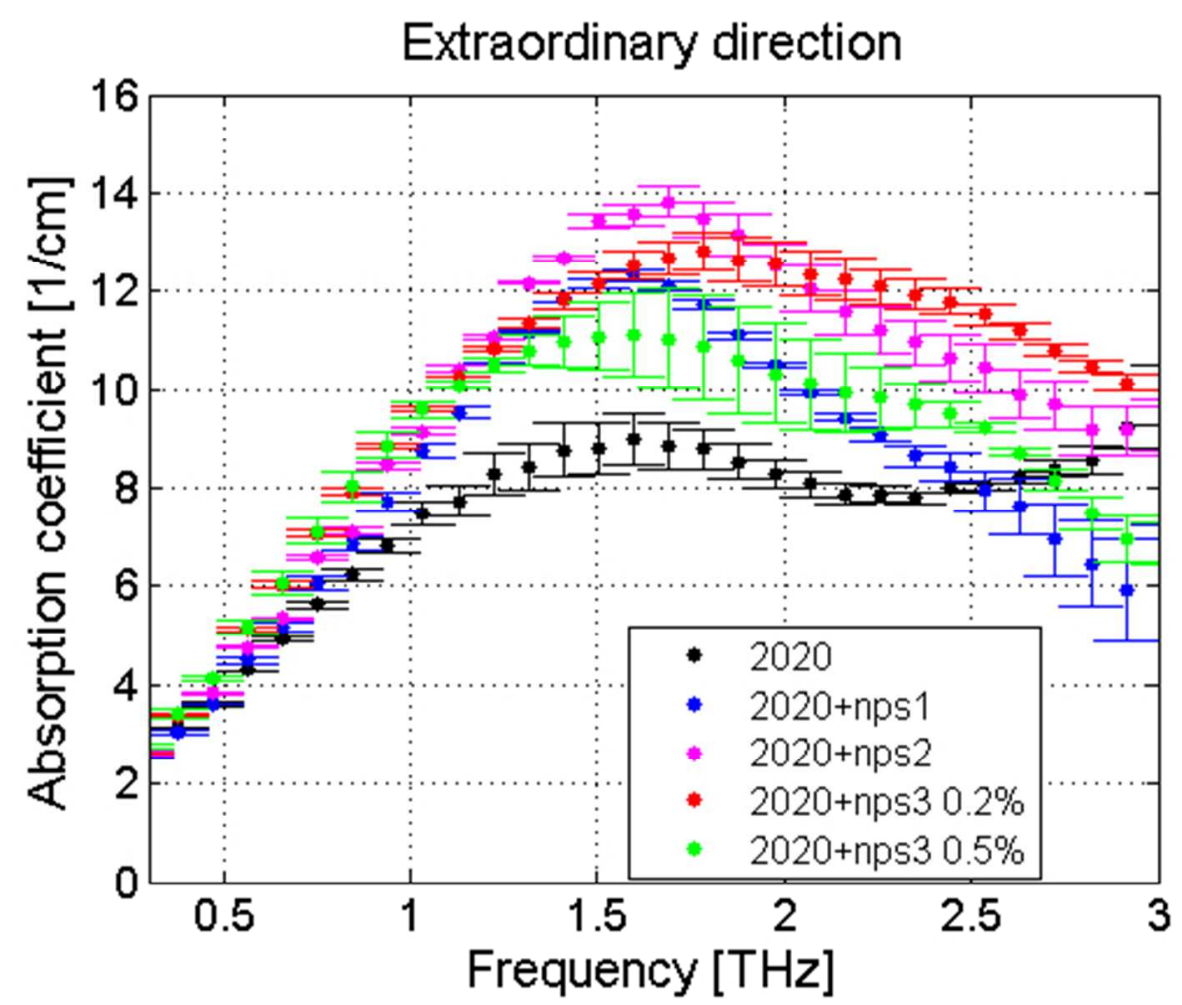

Figure 2d. Extraordinary absorption coefficients of 2020 and its ferroelectric suspensions.

$148 \times 112 \mathrm{~mm}(96 \times 96 \mathrm{DPI})$ 


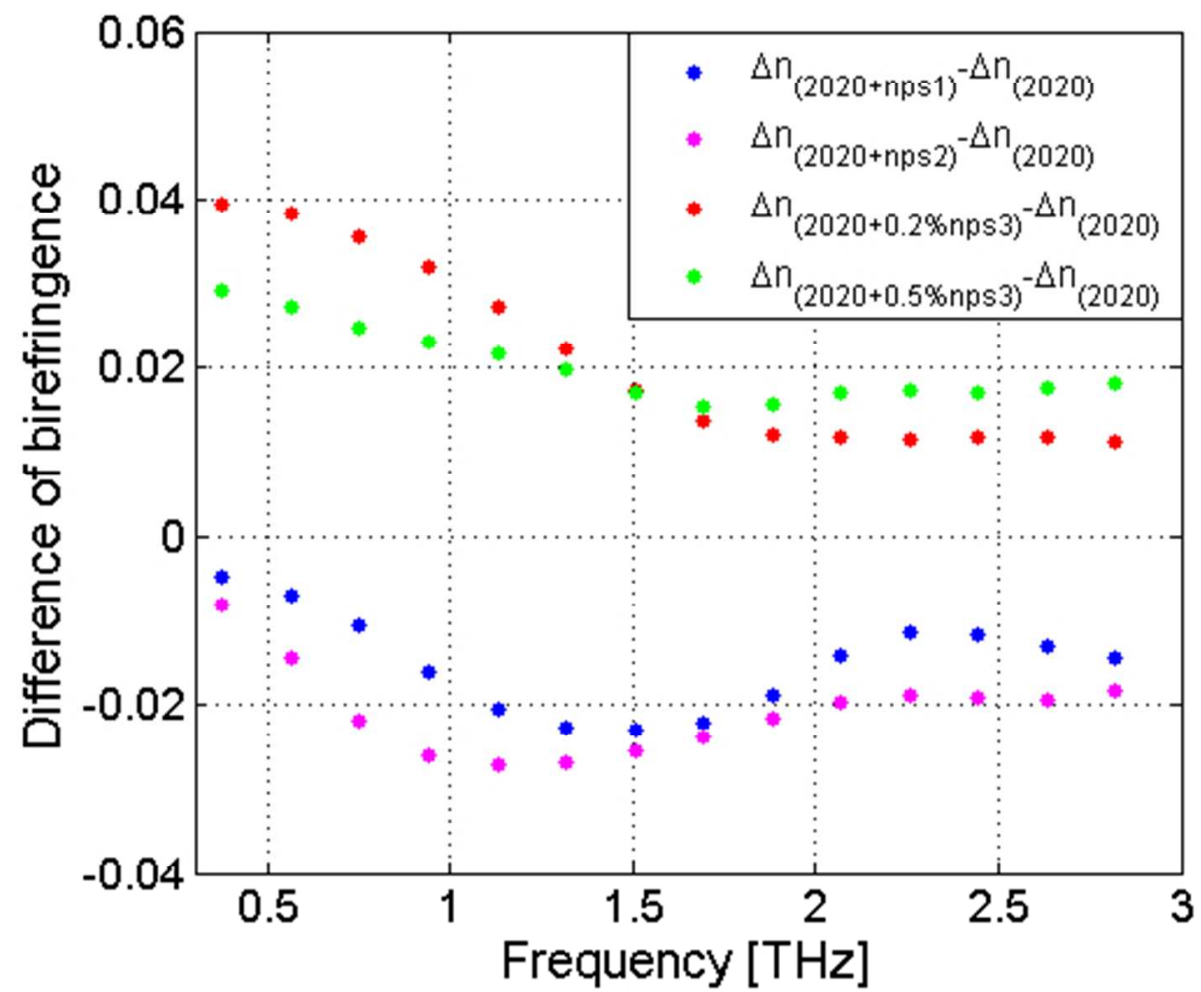

Figure 2e. Difference of birefringence between 2020 and its ferroelectric suspensions.

$148 \times 118 \mathrm{~mm}(96 \times 96 \mathrm{DPI})$ 


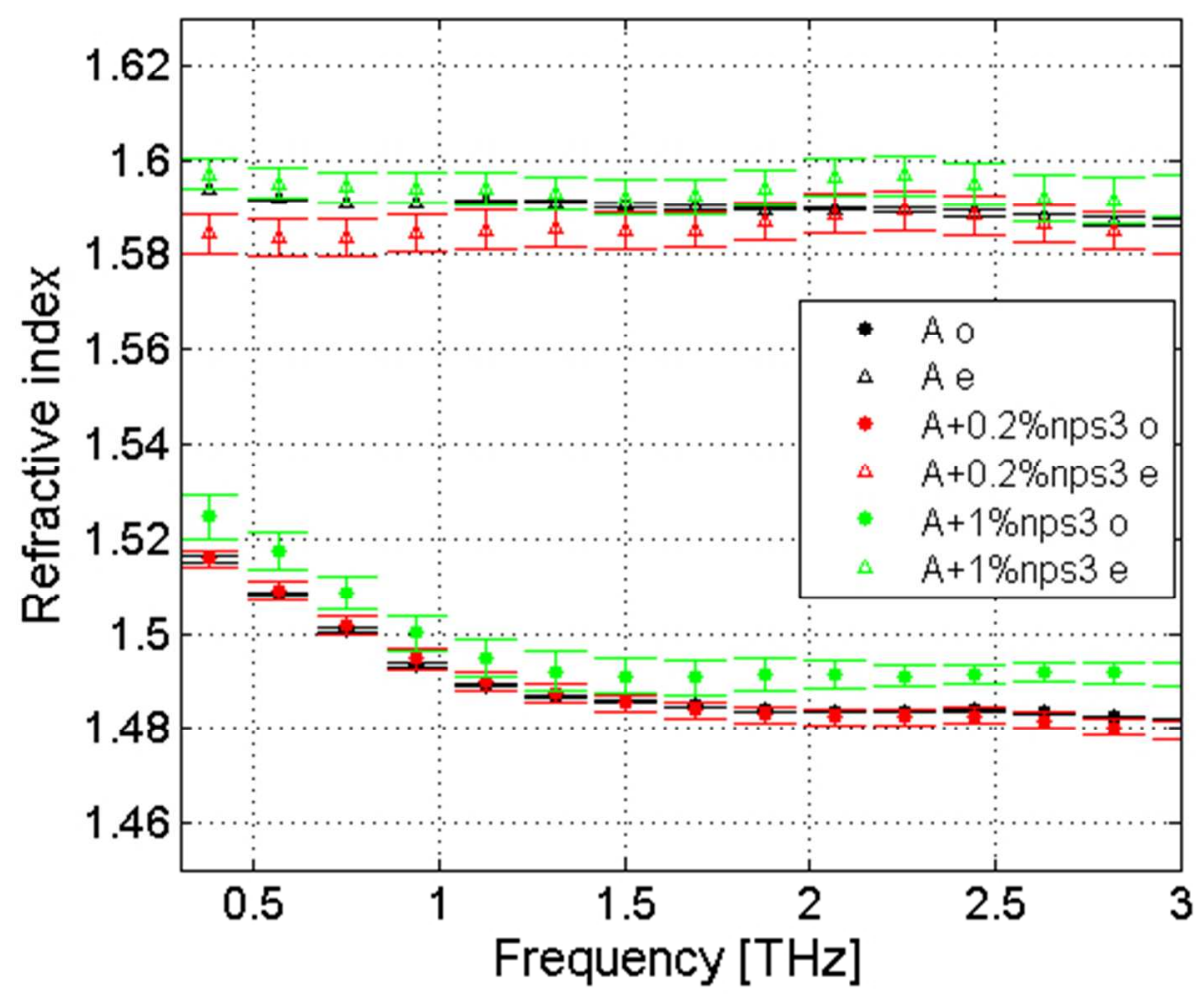

Figure 3a. Refractive indices of compound A and its ferroelectric suspensions.

$148 \times 119 \mathrm{~mm}(96 \times 96 \mathrm{DPI})$ 


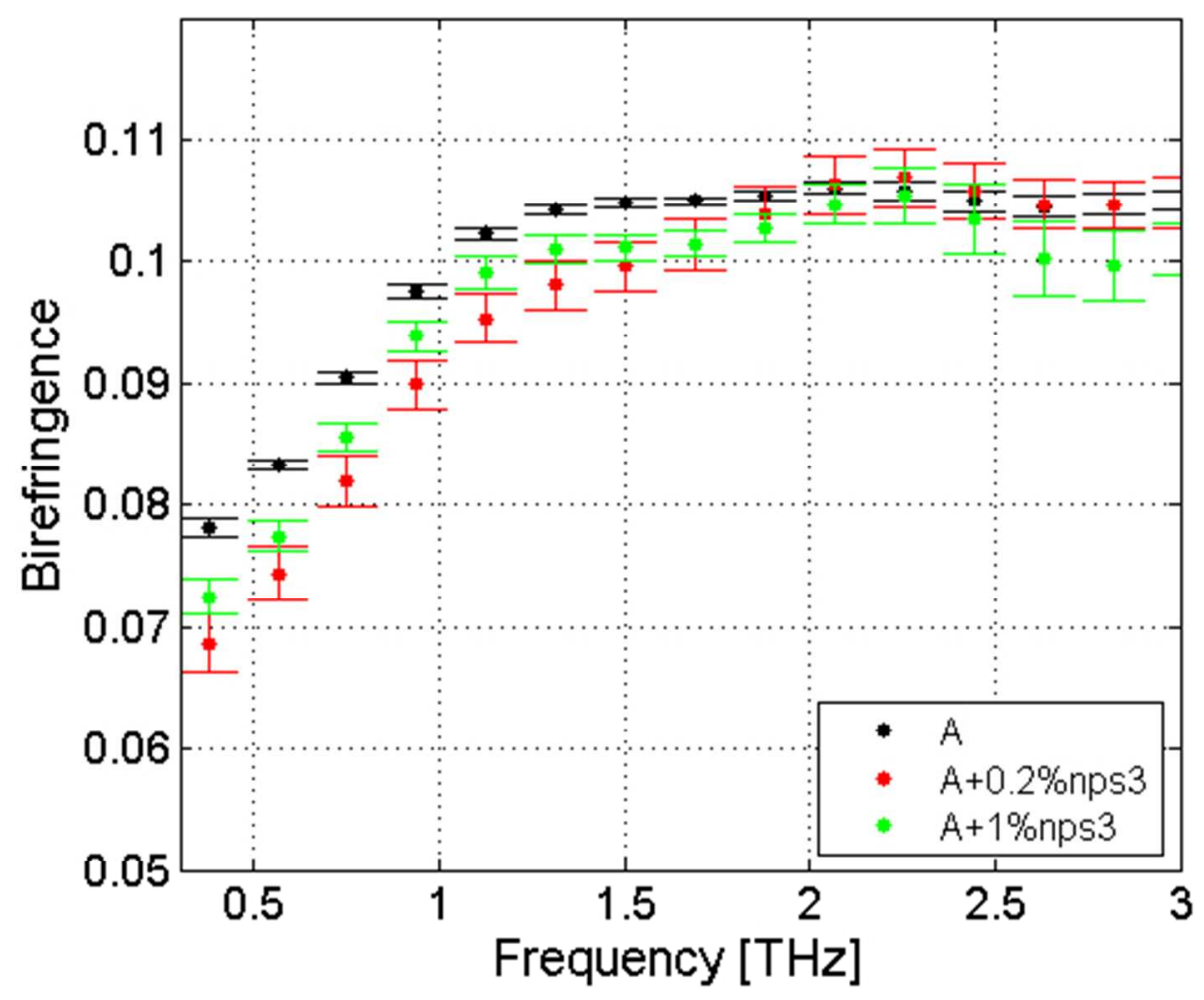

Figure 3b. Birefringence of compound A and its ferroelectric suspensions.

$148 \times 119 \mathrm{~mm}(96 \times 96 \mathrm{DPI})$ 


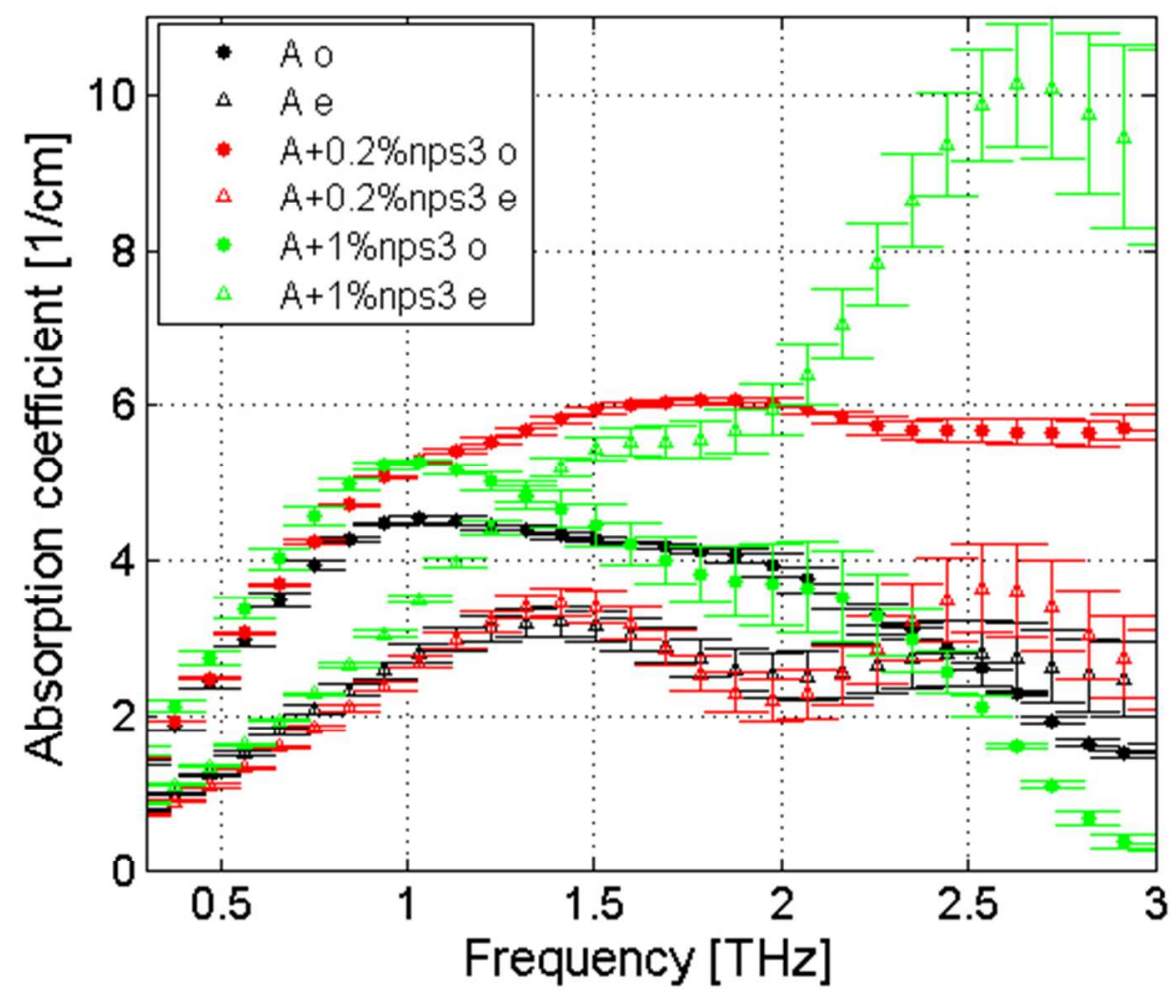

Figure 3c. Absorption coefficients of compound A and its ferroelectric suspensions.

$148 \times 119 \mathrm{~mm}(96 \times 96 \mathrm{DPI})$ 


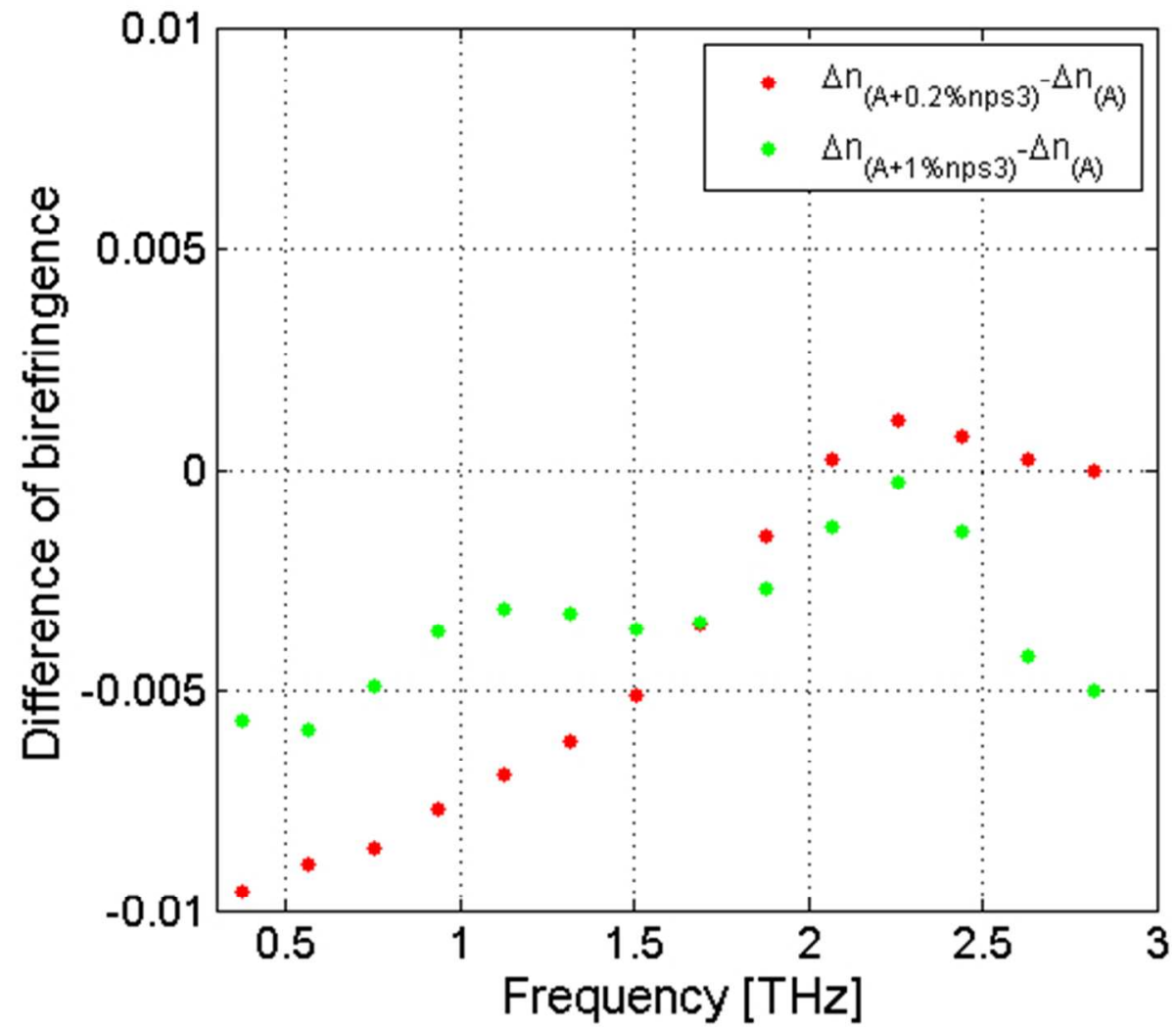

Figure 3d. Difference of birefringence between compound A and its ferroelectric suspensions. $148 \times 125 \mathrm{~mm}(96 \times 96 \mathrm{DPI})$ 


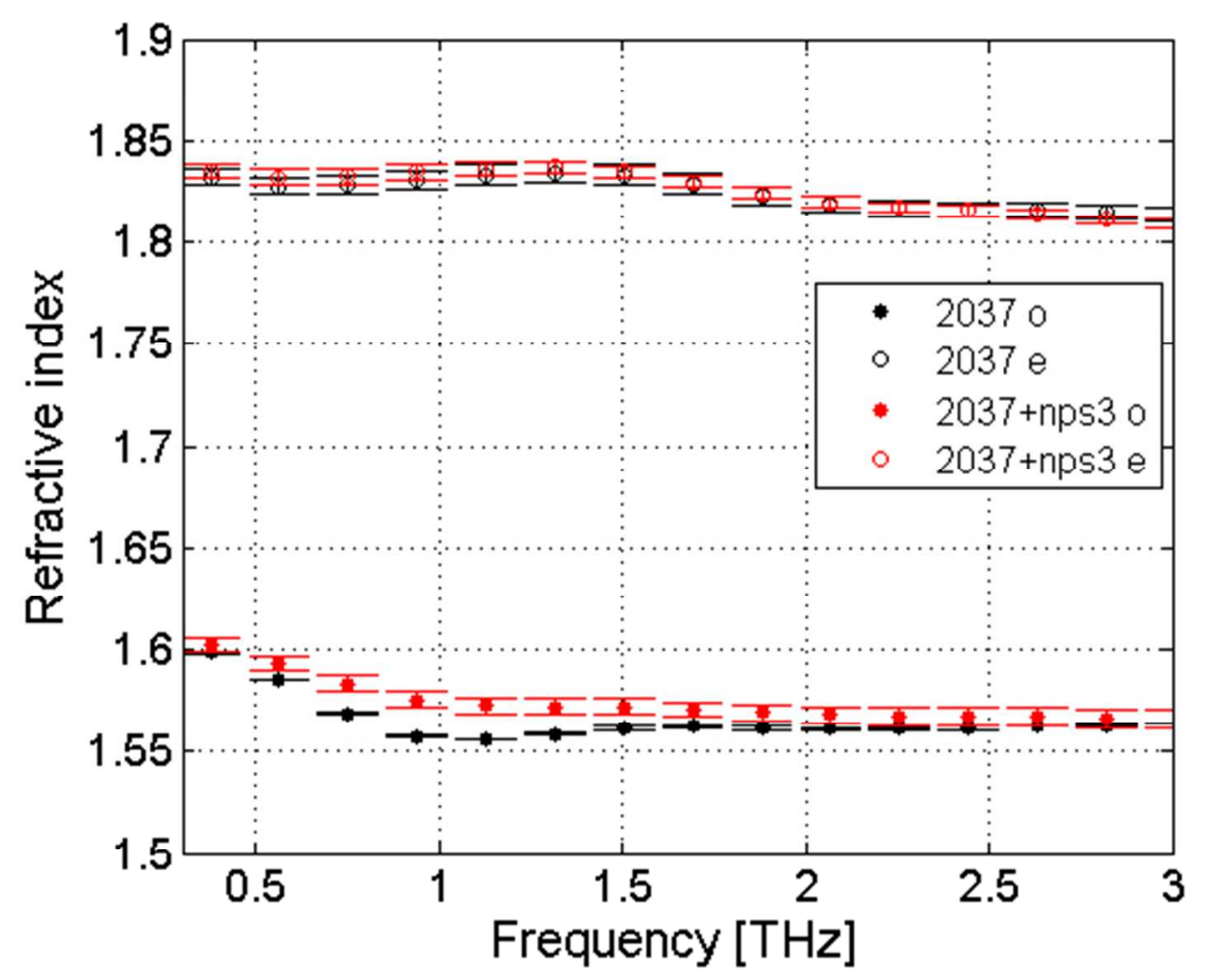

Figure 4a. Refractive indices of 2037 and its ferroelectric suspension. $147 \times 116 \mathrm{~mm}(96 \times 96 \mathrm{DPI})$ 


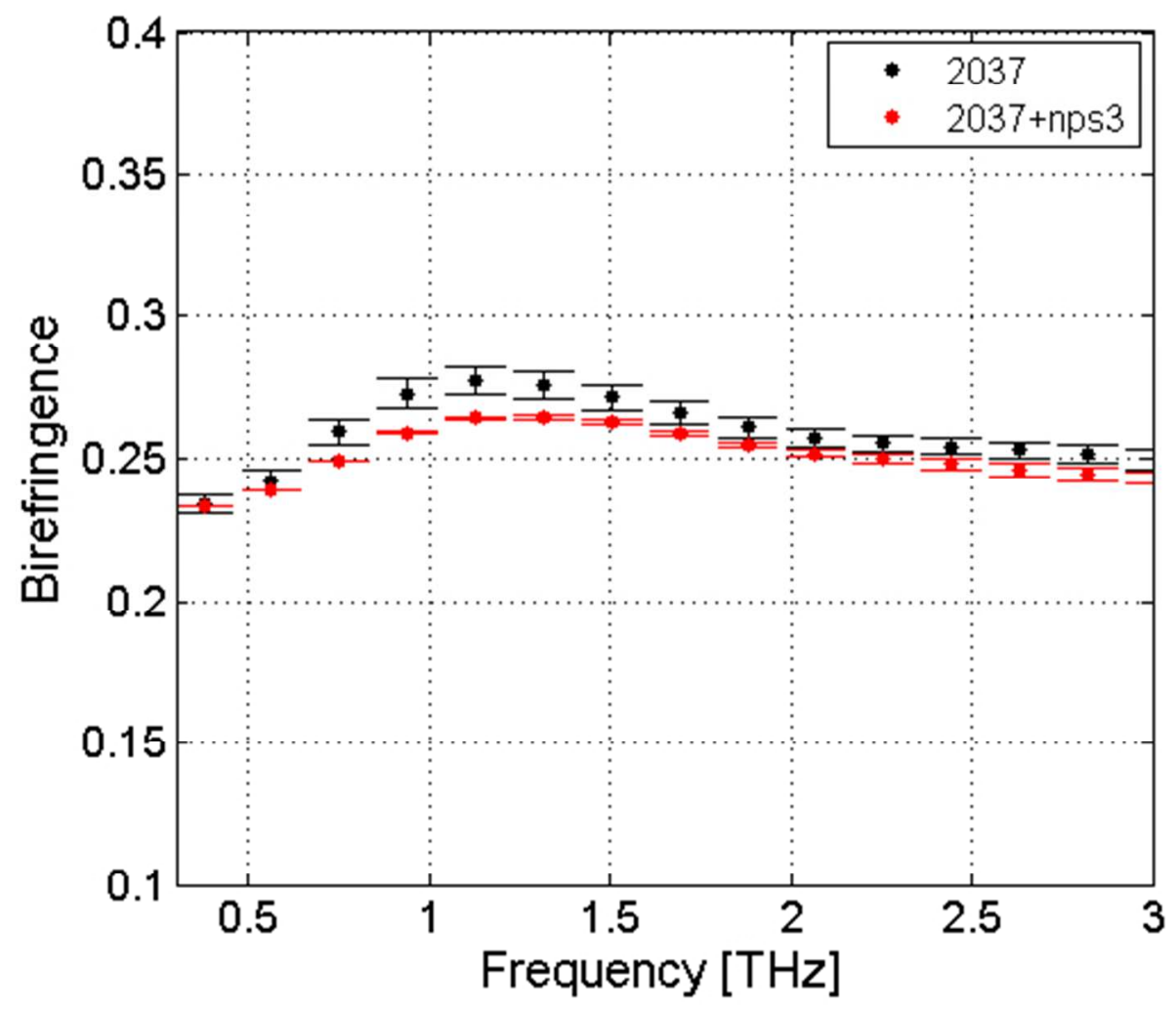

Figure 4b. Birefringence of 2037 and its ferroelectric suspension.

$147 \times 122 \mathrm{~mm}(96 \times 96 \mathrm{DPI})$ 


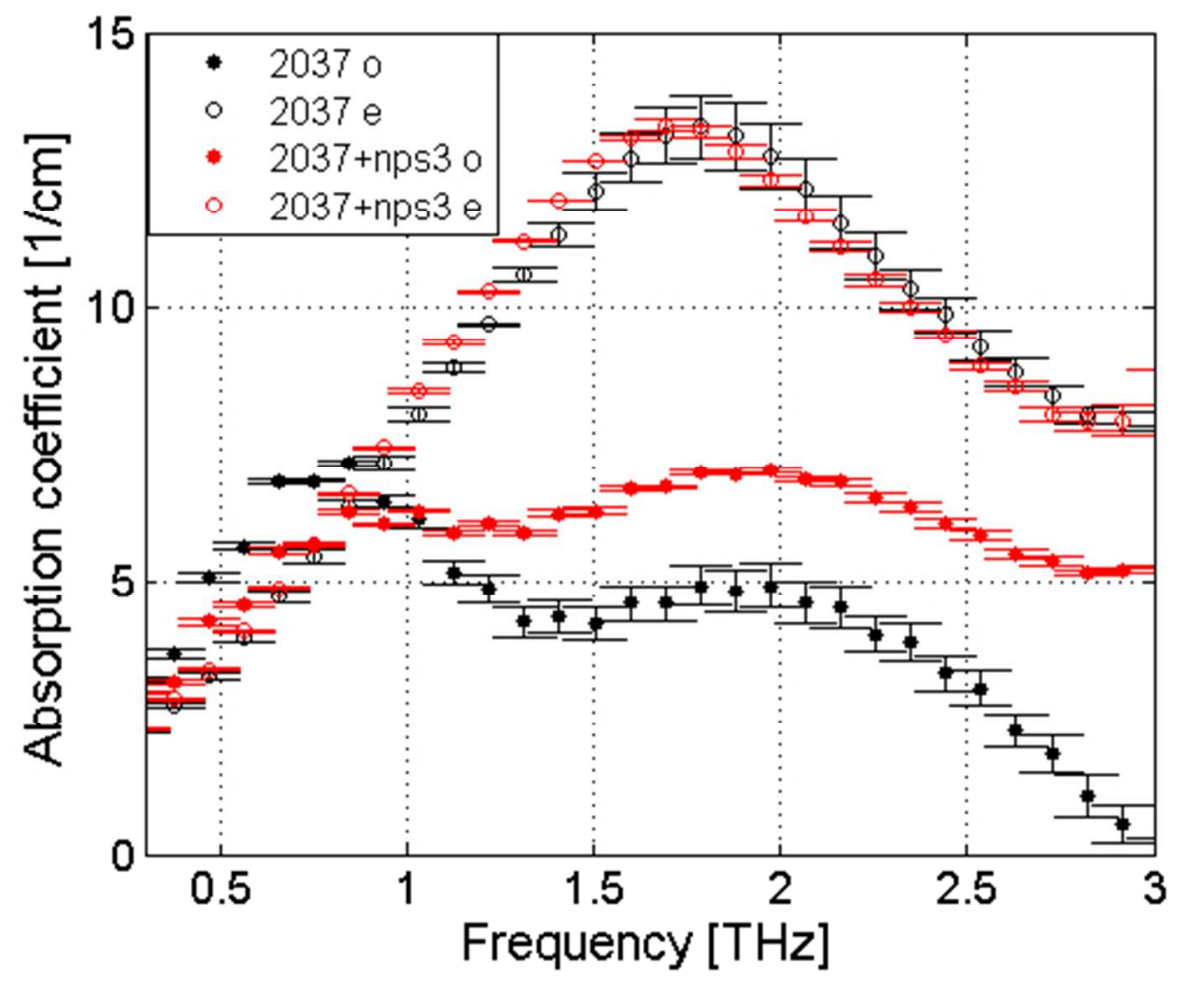

Figure 4c. Absorption coefficients of 2037 and its ferroelectric suspension. $147 \times 116 \mathrm{~mm}(96 \times 96 \mathrm{DPI})$ 


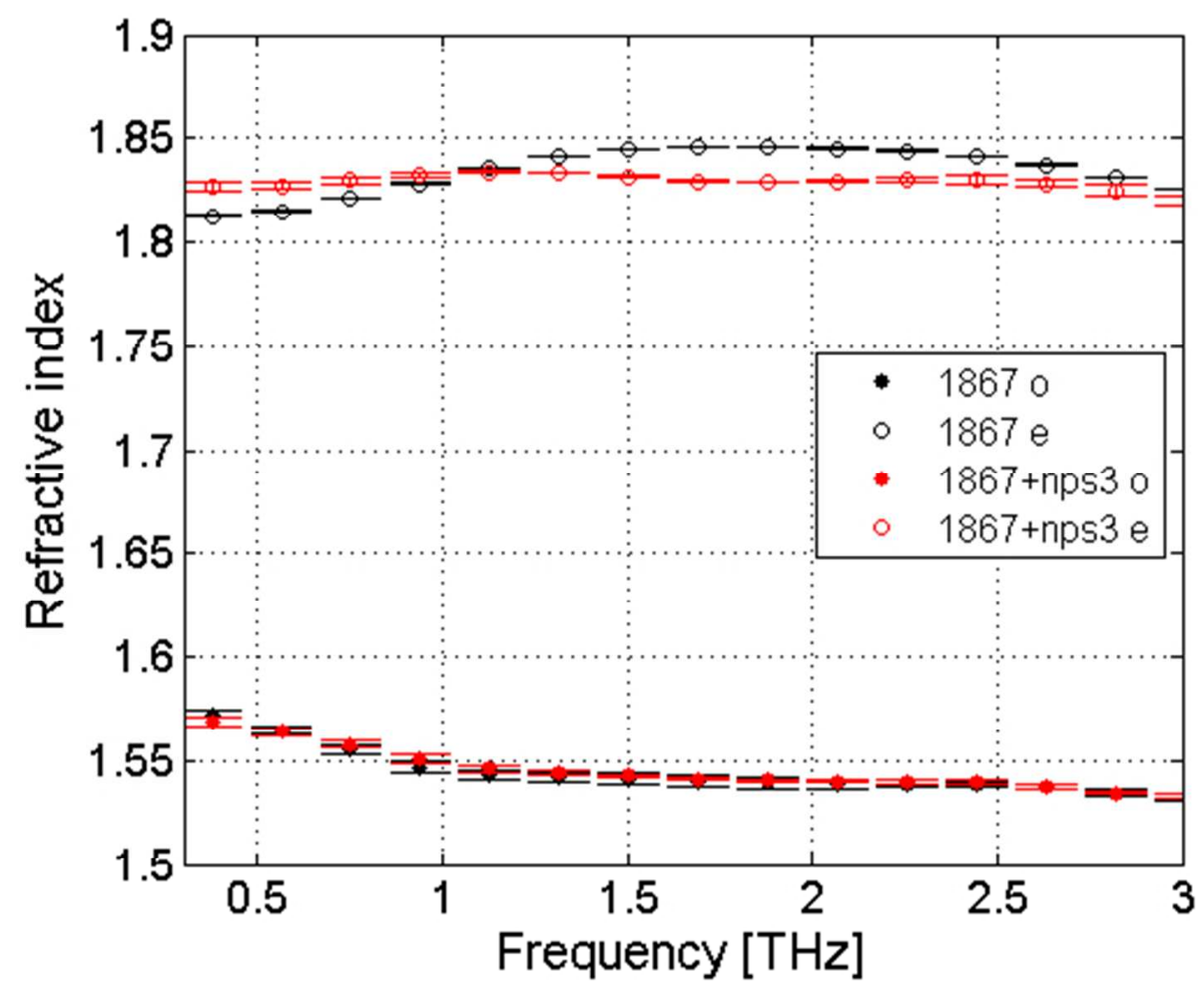

Figure 4d. Refractive indices of 1867 and its ferroelectric suspension.

$148 \times 117 \mathrm{~mm}(96 \times 96$ DPI $)$ 


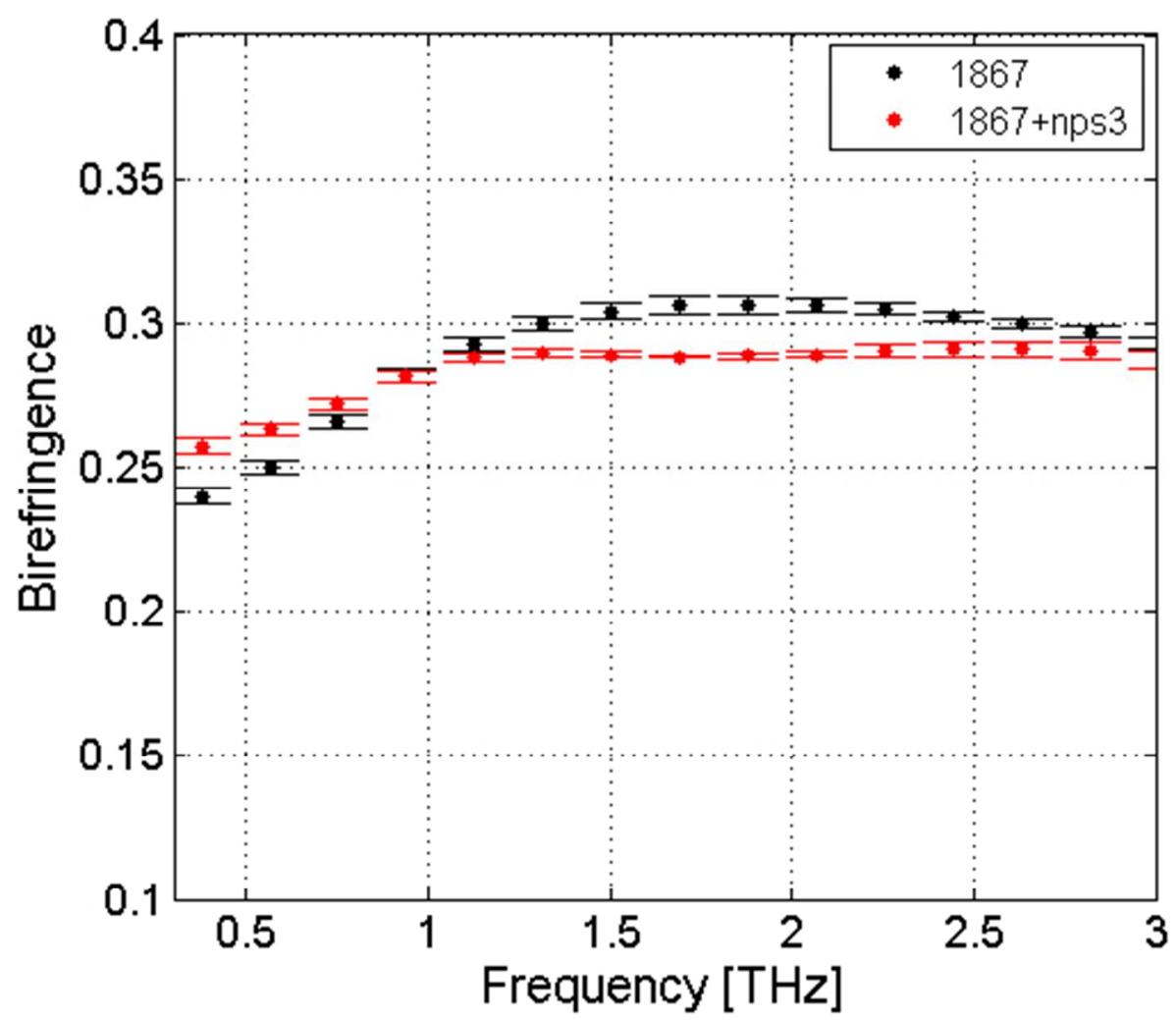

Figure 4e. Birefringence of 1867 and its ferroelectric suspension.

$148 \times 123 \mathrm{~mm}(96 \times 96 \mathrm{DPI})$ 


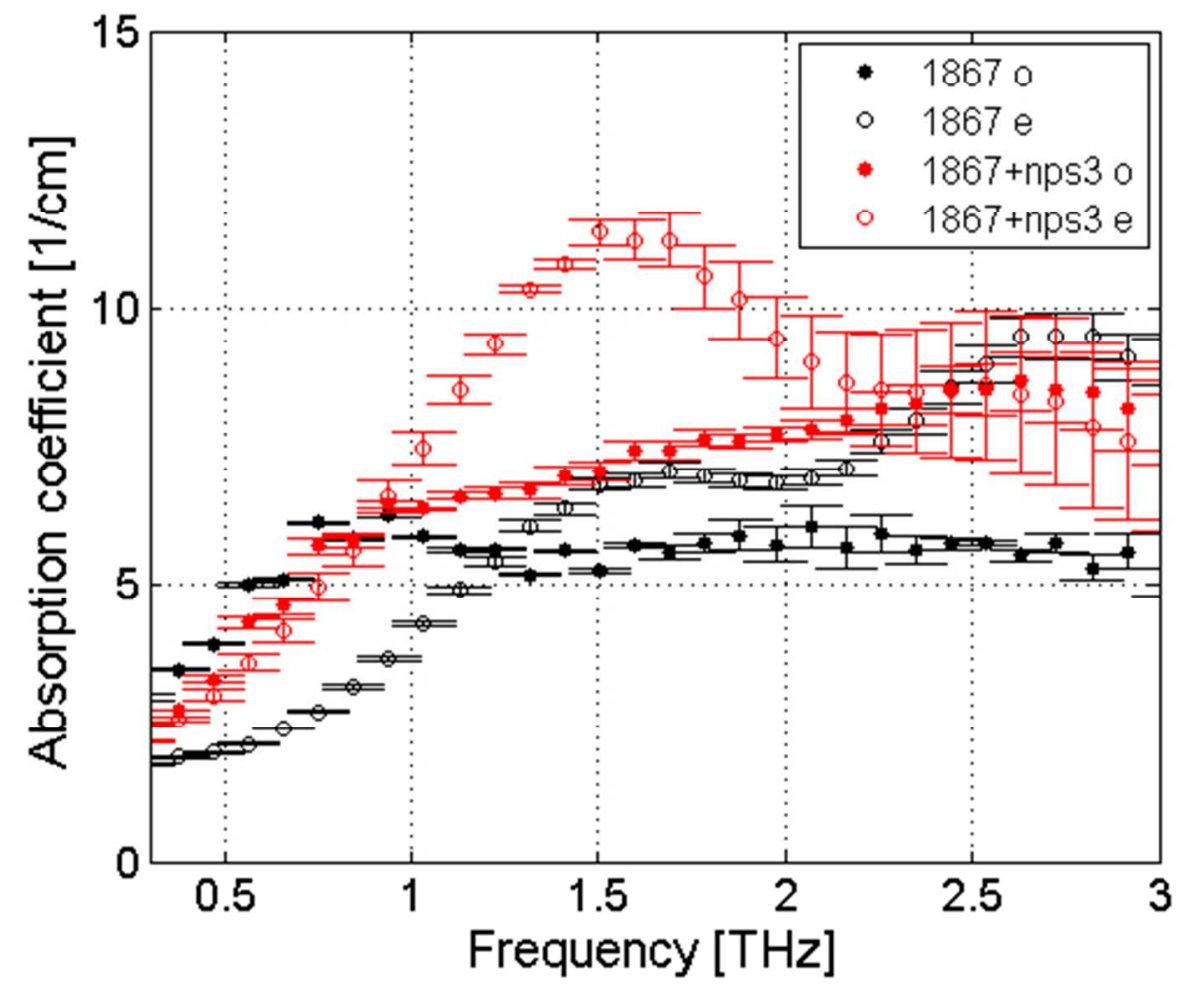

Figure 4f. Absorption coefficients of 1867 and its ferroelectric suspension.

$148 \times 117 \mathrm{~mm}(96 \times 96 \mathrm{DPI})$ 